\title{
Eigenfunctions of Fourier and Fractional Fourier Transforms With Complex Offsets and Parameters
}

\author{
Soo-Chang Pei, Fellow, IEEE, and Jian-Jiun Ding
}

\begin{abstract}
In this paper, we derive the eigenfunctions of the Fourier transform (FT), the fractional FT (FRFT), and the linear canonical transform (LCT) with (1) complex parameters and (2) complex offsets. The eigenfunctions in the cases where the parameters and offsets are real were derived in literature. We extend the previous works to the cases of complex parameters and complex offsets. We first derive the eigenvectors of the offset discrete FT. They approximate the samples of the eigenfunctions of the continuous offset FT. We find that the eigenfunctions of the offset FT with complex offsets are the smoothed Hermite-Gaussian functions with shifting and modulation. Then we extend the results for the case of the offset FRFT and the offset LCT. We can use the derived eigenfunctions to simulate the self-imaging phenomenon for the optical system with energy-absorbing component, mode selection, encryption, and define the fractional $Z$-transform and the fractional Laplace transform.
\end{abstract}

Index Terms-Eigenvalue, eigenvector, fractional Fourier transform (FRFT), fractional Laplace transform, fractional $Z$-transform, linear canonical transform (LCT), offset discrete FT (DFT).

\section{INTRODUCTION}

$\mathbf{I}$ $\mathrm{N}$ THIS PAPER, we discuss the eigenfunctions of the Fourier transform (FT) [1], the fractional FT (FRFT) [2]-[4], and the linear canonical transform (LCT) [5] with complex parameters and complex offset. The offset FT, FRFT, and LCT are defined as follows.

- Offset FT [6], [7]

$$
\begin{aligned}
G_{F}^{\tau, \eta}(\omega) & =\mathrm{FT}_{\tau, \eta}[g(x)] \\
& =\sqrt{1 / 2 \pi} \int_{-\infty}^{\infty} \exp [-j(\omega-\tau)(x-\eta)] g(x) d x .
\end{aligned}
$$

\section{- Offset inverse FT (Offset IFT):}

$$
\begin{aligned}
g(x) & =\operatorname{IFT}_{\tau, \eta}\left[G_{F}^{\tau, \eta}(\omega)\right] \\
& =\sqrt{1 / 2 \pi} \int_{-\infty}^{\infty} \exp [j(x-\eta)(\omega-\tau)] G_{F}^{\tau, \eta}(\omega) d x
\end{aligned}
$$

\footnotetext{
Manuscript received April 22, 2006; revised November 19, 2006. This work was supported by National Science Council, R.O.C., under Contract 93-2219-E002-004 and Contract NSC 93-2752-E-002-006-PAE. This paper was recommended by Associate Editor A. Kummert.

The authors are with the Department of Electrical Engineering, National Taiwan University, Taipei, Taiwan 10617, R.O.C. (e-mail: pei@cc.ee.ntu. edu.tw; djj1@ms63.hinet.net).

Digital Object Identifier 10.1109/TCSI.2007.900182
}

\section{- Offset FRFT:}

$$
\begin{aligned}
G_{\alpha}^{\tau, \eta}(u)= & O_{F}^{\alpha, \tau, \eta}[g(x)] \\
= & \sqrt{\frac{1-j \cot \alpha}{2 \pi}} \exp (j \eta u) \\
& \times \int_{-\infty}^{\infty} \exp \left[\frac{j}{2}(u-\tau)^{2} \cot \alpha-j(u-\tau) x \csc \alpha\right. \\
& \left.\quad+\frac{j}{2} x^{2} \cot \alpha\right] g(x) d x .
\end{aligned}
$$

- Offset LCT [8], [9], [23], [24]:

$$
\begin{aligned}
G_{(a, b, c, d)}^{\tau, \eta}(u)= & O_{F}^{(a, b, c, d, \tau, \eta)}[g(x)] \\
= & \sqrt{\frac{1}{j 2 \pi b}} e^{j \eta u} \\
& \times \int_{-\infty}^{\infty} e^{\frac{j}{2} \frac{d}{b}(u-\tau)^{2}} e^{-j \frac{u-\tau}{b} x} e^{\frac{j}{2} \frac{a}{b} x^{2}} g(x) d x \\
& \text { when } b \neq 0 \\
G_{(a, b, c, d)}^{\tau, \eta}(u)= & O_{F}^{(a, 0, c, d, \tau, \eta)}[g(x)] \\
= & d^{1 / 2} e^{j \eta u+j c d(u-\tau)^{2} / 2} g(d u-d \tau) \\
& \text { when } b=0 .
\end{aligned}
$$

In the above, $a d-b c=1$. In (1)-(5), $\tau$ and $\eta$ are two offset parameters corresponding to displacement and modulation. The parameters $\alpha, a, b, c$, and $d$ in (3), (4) and (5) can be complex. However, from [5]

$$
\operatorname{Im}(a / b) \geq 0 \text { when } a \neq 0, \quad \text { and } \operatorname{Im}(b)=0 \text { when } a=0 .
$$

For the case of the offset FRFT, the above constraint becomes

$$
\operatorname{Im}(\cot \alpha) \geq 0 \text {, i.e., } \operatorname{Im}(\alpha) \leq 0 .
$$

Both the offset FT and the offset FRFT are special cases of the offset LCT

$$
\begin{aligned}
\mathrm{FT}_{\tau, \eta}[g(x)]= & \sqrt{j} e^{-j \tau \eta} O_{F}^{(0,1,-1,0, \tau, \eta)}[g(x)] \\
O_{F}^{\alpha, \tau, \eta}[g(x)]= & \sqrt{\exp (j \alpha)} O_{F}^{(\cos \alpha, \sin \alpha,-\sin \alpha, \cos \alpha, \tau, \eta)} \\
& \times[g(x)] .
\end{aligned}
$$

In addition to the offset FT and FRFT, the offset LCT is also a generalization of many other transforms. For example, when $\{a, b, c, d, \tau, \eta\}=\{1, z \lambda / 2 \pi, 0,1,0,0\}$, the offset LCT becomes the Fresnel transform. When 
$\{a, b, c, d, \tau, \eta\}=\left\{\sigma^{-1}, 0,0, \sigma, 0,0\right\}$, it becomes the scaling operation. When $\{a, b, c, d, \tau, \eta\}=\{1,0,0,1, \tau, \eta\}$, it becomes the shifting and modulation operations. The offset LCT has the following additivity property:

$$
\begin{aligned}
& O_{F}^{\left(a_{2}, b_{2}, c_{2}, d_{2}, \tau_{2}, \eta_{2}\right)}\left\{O_{F}^{\left(a_{1}, b_{1}, c_{1}, d_{1}, \tau_{1}, \eta_{1}\right)}[f(x)]\right\} \\
&=\exp (j \phi) O_{F}^{(e, f, g, h, r, s)}[f(x)]
\end{aligned}
$$

where

$$
\begin{aligned}
{\left[\begin{array}{ll}
e & f \\
g & h
\end{array}\right] } & =\left[\begin{array}{ll}
a_{2} & b_{2} \\
c_{2} & d_{2}
\end{array}\right]\left[\begin{array}{ll}
a_{1} & b_{1} \\
c_{1} & d_{1}
\end{array}\right] \\
{\left[\begin{array}{l}
r \\
s
\end{array}\right] } & =\left[\begin{array}{ll}
a_{2} & b_{2} \\
c_{2} & d_{2}
\end{array}\right]\left[\begin{array}{l}
\tau_{1} \\
\eta_{1}
\end{array}\right]+\left[\begin{array}{l}
\tau_{2} \\
\eta_{2}
\end{array}\right]
\end{aligned}
$$

and $\phi$ is some phase defined in [10].

The eigenfunctions of the nonoffset FT and FRFT are known to be Hermite-Gaussian functions [1], [3]. The eigenfunctions of the nonoffset LCT were derived in [10], [11]. In [12], we found that the eigenfunctions of the offset FT, FRFT, LCT have the following form:

$$
\begin{array}{r}
E_{(a, b, c, d, \tau, \eta)}(x)=\exp \left[j \frac{c \tau+}{2-a-d} x\right] E_{(a, b, c, d)} \\
\times\left(x-\frac{(1-d) \tau+b \eta}{2-a-d}\right)
\end{array}
$$

where $E_{(a, b, c, d)}(x)$ is the eigenfunction of the nonoffset FT, FRFT, and LCT. However, the results in [12] are suitable for the case whether the parameters and the offsets are real. In the case where $\{a, b, c, d, \tau, \eta\}$ are complex, there are two problems.

(1) In (11), $[(1-d) \tau+b \eta](2-a-d)^{-1}$ may be complex and it is very hard to define the displacement with complex distance.

(2) The eigenfunction $E_{a, b, c, d}(x)$ of the nonoffset LCT with complex $\{a, b, c, d\}$ has not been found completely. In [25], Wolf derived the eigenvectors of the complex LCT in some special cases (including the Fourier, Hankel, bilateral Laplace, Bargmann, Weierstrass-Gauss, and Barut-Girardello transforms), however, the general result was not derived. They find the functions that satisfy

$O_{F}^{(a, b, c, d)}(\phi(x))=C \exp \left(j \alpha x^{2}+j \beta x\right) \phi(\gamma x+\delta)$

but the eigenfunction that satisfies

$$
O_{F}^{(a, b, c, d)}(\phi(x))=\lambda \phi(x)
$$

and

$$
O_{F}^{(a, b, c, d, \tau, \eta)}(\phi(x))=\lambda \phi(x)
$$

was not found yet.

Due to the above two facts, we cannot use (11) to find the eigenfunctions of the offset FT, FRFT, and LCT with complex parameters and offset in all the cases.

In this paper, we will derive the eigenfunctions of the offset FT, FRFT, and LCT for all the cases, including the cases where the offsets $\tau, \eta$ and the parameters $\alpha, a, b, c$, and $d$ are complex. The results are useful for analyzing the self-image and the resonance phenomena for the optical system with the decayed component (i.e., the component that can absorb light energy).

\section{EIGENVECTORS OF DFT COMPLEX OfFSETS}

\section{A. Previous Works About Eigenvectors of Offset DFT}

Before deriving the eigenfunctions of the offset FT, we first discuss the eigenvectors of its discrete counterpart, i.e., the offset discrete FT (offset DFT), which is defined as [6], [7]

$$
\begin{aligned}
X_{p, q}[m] & =\operatorname{DFT}_{p, q}(x[n]) \\
& =\sqrt{N^{-1}} \sum_{n=0}^{N-1} \exp \left[-j \frac{2 \pi}{N}(m-p)(n-q)\right] x[n] \\
n, m & \in[0, N-1] .
\end{aligned}
$$

It generalizes DFT by two extra parameters, $p$ and $q$. It extends the utilities of the DFT and is useful for filter design, signal analysis, and the fast algorithm development [6], [7]. When $p$ or $q$ is complex

$$
p=\mu+j \sigma, \quad q=\kappa+j \rho, \quad \text { where } \mu, \sigma, \kappa, \text { and } \rho \text { are real }
$$

the offset DFT becomes the DFT with the real offset terms $(\mu, \kappa)$ and the decayed terms $\exp (-2 \pi \sigma n / N)$ and $\exp (-2 \pi \rho m / N)$

$$
\begin{aligned}
& e^{-j 2 \pi \sigma \rho / N-2 \pi(\kappa \sigma+\mu \rho) / N} X_{\mu+j \sigma, \kappa+j \rho}[m] \\
& \quad=N^{-1 / 2} e^{\frac{-2 \pi \rho m}{N}} \sum_{n=0}^{N-1} e^{\frac{-j 2 \pi(m-\mu)(n-\kappa)}{N}} e^{\frac{-2 \pi \sigma n}{N}} x[n] .
\end{aligned}
$$

The eigenvector of the original DFT (i.e., $p=q=0$ ) has been widely discussed [13]. In [14], Tseng derive the eigenvectors of the offset DFT for the special case of $p=q=1 / 2$. In [15], Pei and Ding found that, when $p+q=$ integer, the eigenvectors of the offset DFT could be derived from the methods of 1) sampling and summing the eigenfunctions of the continuous counterpart; 2) commutative matrix; or 3) linear combination. However, in [15], the way to derive the eigenvector when $p+q$ is not an integer was not found yet. In [16], Clary and Mugler extended Grünbaum's work [17] and found that, when $p=q$, the following tridiagonal matrix can commute with the offset DFT matrix:

$$
\begin{aligned}
T_{p}[n, n] & =-2 \cos \left[\frac{\pi(2 p+1)}{N}\right] \sin \left[\frac{\pi n}{N}\right] \sin \left[\frac{\pi(2 p-n)}{N}\right] \\
T_{p}[n, n-1] & =T_{p}[n-1, n]=\sin [\pi n / N] \sin [\pi(2 p+1-n) / N] \\
T_{p}[m, n] & =0, \quad \text { otherwise. }
\end{aligned}
$$

Since $\mathbf{T}_{\mathbf{p}}$ commutes with the offset DFT matrix when $p=q$, we can use it to determine the eigenvectors of the offset DFT for the case where $p=q$.

In the next subsection, we extend the previous works and find the eigenvectors of the offset DFT with arbitrary $\boldsymbol{p}$ and $\boldsymbol{q}$, including the cases where $p+q \neq$ integer, where $p \neq q$, and where $p, q$, or both of them are complex numbers. If the number of points $N$ is sufficiently large, the eigenvectors offset DFT 
derived in this section will approximate to the samples of the eigenfunctions of the continuous offset FT.

\section{B. Generalized DFT Eigenvectors and Eigenvalues}

When $p+q \neq$ integer, the eigenvectors of the offset DFT can be determined from the following tridiagonal matrix:

$$
\mathbf{T}_{\mathbf{p}, \mathbf{q}}=\left[\begin{array}{ccccccc}
D_{0} & C_{1} & 0 & 0 & \cdots & 0 & 0 \\
B_{1} & D_{1} & C_{2} & 0 & \cdots & 0 & 0 \\
0 & B_{2} & D_{2} & C_{3} & \ddots & \vdots & \vdots \\
0 & 0 & B_{3} & \ddots & \ddots & 0 & \vdots \\
\vdots & \vdots & 0 & \ddots & \ddots & C_{N-2} & 0 \\
0 & \vdots & \vdots & \cdots & B_{N-2} & D_{N-2} & C_{N-1} \\
0 & 0 & 0 & \cdots & 0 & B_{N-1} & D_{N-1}
\end{array}\right]
$$

$$
\begin{aligned}
& B_{n}=e^{j \pi(q-p) / N} \sin [\pi n / N] \sin [\pi(p+q+1-n) / N] \\
& C_{n}=e^{j \pi(p-q) / N} \sin [\pi n / N] \sin [\pi(p+q+1-n) / N] \\
& D_{n}=-2 \cos \left[\frac{\pi(p+q+1)}{N}\right] \sin \left[\frac{\pi n}{N}\right] \sin \left[\frac{\pi(p+q-n)}{N}\right] .
\end{aligned}
$$

(a) The matrix $\mathbf{T}_{\mathbf{p}, \mathbf{q}}$ commutes with the offset DFT matrix $\mathbf{F}_{\mathrm{p}, \mathrm{q}}$ (i.e., $\mathbf{T}_{\mathrm{p}, \mathrm{q}} \mathbf{F}_{\mathrm{p}, \mathrm{q}}=\mathbf{F}_{\mathrm{p}, \mathrm{q}} \mathbf{T}_{\mathrm{p}, \mathrm{q}}$ )

Proof: If $\mathbf{G}=\mathbf{T}_{\mathbf{p}, \mathbf{q}} \mathbf{F}_{\mathbf{p}, \mathbf{q}}$ and $\mathbf{H}=\mathbf{F}_{\mathbf{p}, \mathbf{q}} \mathbf{T}_{\mathbf{p}, \mathbf{q}}$, then

$$
\begin{aligned}
& e^{j \frac{2 \pi}{N}(m-p)(n-q)} G[m, n] \\
& =e^{j \frac{\pi}{N}(2 n-p-q)} \sin \left[\frac{\pi m}{N}\right] \sin \left[\frac{\pi(p+q+1-m)}{N}\right] \\
& -2 \cos [\pi(p+q+1) / N] \sin [\pi m / N] \\
& \times \sin [\pi(p+q-m) / N]+e^{-j \pi(2 n-p-q) / N} \\
& \times \sin [\pi(m+1) / N] \sin [\pi(p+q-m) / N] \\
& =-\cos [\pi(p+q-2 n) / N] \cos [\pi(p+q+1) / N] \\
& +e^{j \pi / N} \cos \left[\frac{2 \pi}{N}(n-m)\right] / 2 \\
& +e^{-j \pi / N} \cos \left[\frac{2 \pi}{N}(m+n-p-q)\right] / 2 \\
& +\cos \left[\frac{\pi}{N}(p+q+1)\right]\left\{\cos \left[\frac{\pi}{N}(p+q)\right]\right. \\
& -\cos \left[\frac{\pi}{N}(p+q-2 m)\right] \\
& e^{j \frac{2 \pi}{N}(m-p)(n-q)} H[m, n] \\
& =e^{j \frac{\pi}{N}(2 m-p-q)} \sin \left[\frac{\pi n}{N}\right] \sin \left[\frac{\pi(p+q+1-n)}{N}\right] \\
& -2 \cos [\pi(p+q+1) / N] \sin [\pi n / N] \\
& \times \sin [\pi(p+q-n) / N]+e^{-j \pi(2 m-p-q) / N} \\
& \times \sin [\pi(n+1) / N] \sin [\pi(p+q-n) / N] \text {. }
\end{aligned}
$$

After some calculation, we obtain

$$
\begin{aligned}
& e^{j \frac{2 \pi}{N}(m-p)(n-q)} H[m, n]=e^{j \frac{2 \pi}{N}(m-p)(n-q)} G[m, n] \\
& \mathbf{T}_{\mathbf{p}, \mathbf{q}} \mathbf{F}_{\mathbf{p}, \mathbf{q}}=\mathbf{F}_{\mathbf{p}, \mathbf{q}} \mathbf{T}_{\mathbf{p}, \mathbf{q}} \cdot \#
\end{aligned}
$$

(b) When $p+q \neq$ integer all the eigenvalues of $\mathbf{T}_{\mathbf{p}, \mathbf{q}}$ are distinct.
Thus, if $\mathbf{v}$ is an eigenvector of $\mathbf{T}_{\mathbf{p}, \mathbf{q}}$, (i.e., $\mathbf{T}_{\mathbf{p}, \mathbf{q}} \cdot \mathbf{v}=\tau \cdot \mathbf{v}$ ), then

$$
\mathbf{T}_{\mathbf{p}, \mathbf{q}}\left[\mathbf{F}_{\mathbf{p}, \mathbf{q}} \cdot \mathbf{v}\right]=\mathbf{F}_{\mathbf{p}, \mathbf{q}} \mathbf{T}_{\mathbf{p}, \mathbf{q}} \cdot \mathbf{v}=\mathbf{F}_{\mathbf{p}, \mathbf{q}} \tau \cdot \mathbf{v}=\tau \cdot\left[\mathbf{F}_{\mathbf{p}, \mathbf{q}} \cdot \mathbf{v}\right] \text {. }
$$

In other words, $\mathbf{F}_{\mathbf{p}, \mathbf{q}} \cdot \mathbf{v}$ is also an eigenvector of $\mathbf{T}_{\mathbf{p}, \mathbf{q}}$ corresponding to the same eigenvalue. Since the eigenvalues of $\mathbf{T}_{\mathbf{p}, \mathbf{q}}$ are distinct, the eigenspace-dimension corresponding to $\tau$ should be 1 . Thus, we can conclude that $\mathbf{F}_{\mathbf{p}, \mathbf{q}} \cdot \mathbf{v}$ must be a multiple of $\mathbf{v}$ (i.e., $\mathbf{F}_{\mathbf{p}, \mathbf{q}} \cdot \mathbf{v}=\lambda \mathbf{v}$ for some constant $\lambda$ ). Thus, $\mathbf{v}$ is also an eigenvector of $\mathbf{F}_{\mathbf{p}, \mathbf{q}}$. That is,

- The eigenvectors of $T_{p, q}$ are also the eigenvectors of $F_{p, q}$ when $p+q \neq$ integer.

Therefore, to find the eigenvectors of the offset DFT when $p+q \neq$ integer, we can first find the eigenvalues $\tau_{p}$ 's of $\mathbf{T}_{\mathbf{p}, \mathbf{q}}$ (but they are not the eigenvalues of $\mathbf{F}_{\mathbf{p}, \mathbf{q}}$ )

$$
\operatorname{det}\left(\mathbf{T}_{\mathbf{p}, \mathbf{q}}-\tau_{p} \mathbf{I}\right)=0, \quad p=0 \sim N-1 .
$$

After $\tau_{p}$ 's are determined, the eigenvectors of $\mathbf{T}_{\mathbf{a}, \mathbf{b}}$ can be computed from the recursive process of

$$
\begin{aligned}
\mathbf{v}_{\mathbf{p}}[0] & =\text { any constant, } \quad \mathbf{v}_{\mathbf{p}}[1]=\left(\tau_{p}-D_{0}\right) \mathbf{v}_{\mathbf{p}}[0] / C_{1} \\
\mathbf{v}_{\mathbf{p}}[n] & =\left(\tau_{p}-D_{n-1}\right) \mathbf{v}_{\mathbf{p}}[n-1] / C_{n}-B_{n-1} \mathbf{v}_{\mathbf{p}}[n-2] / C_{n} \\
& \text { for } n=2 \sim N-1 .
\end{aligned}
$$

The eigenvectors $\mathbf{v}_{\mathbf{p}}[n]$ we obtained are also the eigenvectors of the offset DFT when $p+q \neq$ integer. We can sort the eigenvectors $\mathbf{v}_{\mathbf{p}}[n]$ according to the amplitudes of $\tau_{p}$ 's, i.e.,

$$
\left|\tau_{0}\right|>\left|\tau_{1}\right|>\left|\tau_{2}\right|>\ldots>\left|\tau_{N-1}\right| \text {. }
$$

When $p+q$ is an integer, the matrix $\mathbf{T}_{\mathbf{p}, \mathbf{q}}$ may have repeated eigenvalues and we can not use it to determine the eigenvectors of $\mathbf{F}_{\mathbf{p}, \mathbf{q}}$. In this case, we can apply the methods introduced in [15] to determine the eigenvectors of the offset DFT.

Using the method in (18)-(23) (for $p+q=$ integer) together with the works in [15] (for $p+q \neq$ integer), we can determine the eigenvectors of the offset DFT with arbitrary offset, including the case where $p$ and $q$ are complex numbers (i.e., the decayed offset DFT defined in (16)). The proposed method is more general than the works in [14]-[17].

\section{EIgENFunctions of FTs With COMPLEX OfFSETS}

Comparing (1) with (14), it can be seen that the relations between the variables and the parameters of the offset DFT and the offset FT are

$$
\begin{aligned}
x & =\sqrt{\Delta}\left(n-N_{1}\right), \omega=\sqrt{\Delta}\left(m-N_{1}\right), \tau=\sqrt{\Delta}\left(p-N_{1}\right) \\
\eta & =\sqrt{\Delta}\left(q-N_{1}\right)
\end{aligned}
$$$$
\text { where } \Delta=2 \pi / N \text { and } N_{1}=(N-1) / 2 \text {. }
$$

(In (24), we do the subtractions of $2^{-1}(N-1)$ to centralize the position of $x=0$ ). Note that

$$
p=\tau \sqrt{\Delta^{-1}}+N_{1}, \quad q=\eta \sqrt{\Delta^{-1}}+N_{1} .
$$


(a)

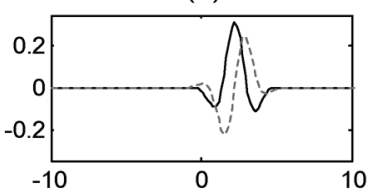

(c)

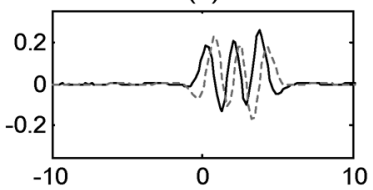

(e)

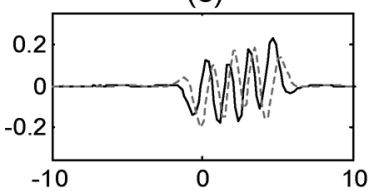

(b)

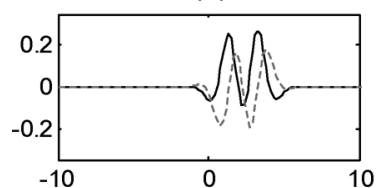

(d)

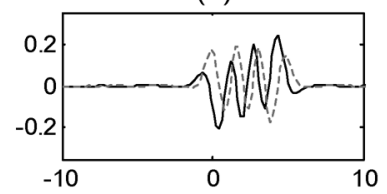

(f)

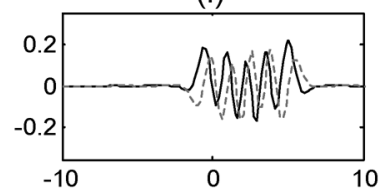

Fig. 1. First six eigenfunctions of the offset FT where $\tau=0.6+0.25 j$ and $\eta=3.9+0.19 j$. The solid lines are real part and the dash lines are imaginary parts. (a) $E_{0}(x)$. (b) $E_{1}(x)$. (c) $E_{2}(x)$. (d) $E_{3}(x)$. (e) $E_{4}$ (x). (f) $E_{5}$ (x). (a)

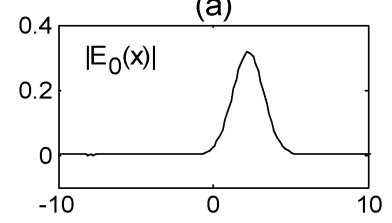

(c)

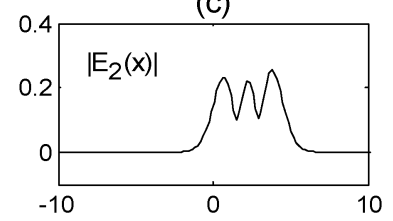

(e)

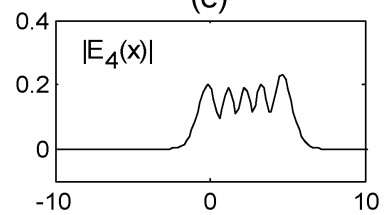

(b)

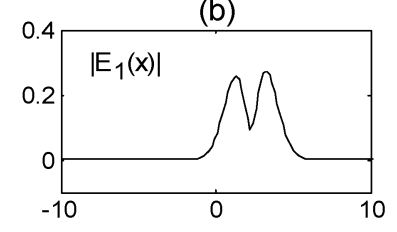

(d)

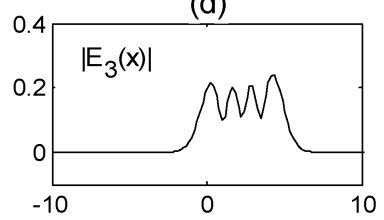

(f)

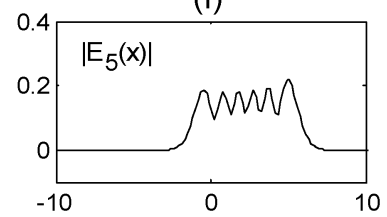

Fig. 2. Amplitudes of the eigenfunctions in Fig. 1.

Therefore, suppose that $v[n]$ is an eigenvectors of the DFT with offsets $\left\{\tau \Delta^{-0.5}+N_{1}, \eta \Delta^{-0.5}+N_{1}\right\}$

$$
\operatorname{DFT}_{\tau \Delta^{-0.5}+N_{1}, \eta \Delta^{-0.5}+N_{1}}(v[n])=\lambda v[n]
$$

then $v[n]$ approximates the samples of the eigenfunction of the offset FT with offsets $\{\tau, \eta\}$

$$
\left.v[n] \approx E(x)\right|_{x=\sqrt{\Delta}\left(n-N_{1}\right)} \text { where } \mathrm{FT}_{\tau, \eta}[E(x)]=\lambda E(x) .
$$

If $N$ is sufficient large, then the approximation in (27) is very accurate and we can use the commuting matrix in (18) together with (21) and (22) to derive the eigenfunctions of the offset FT.

In Figs. 1 and 2, we give some examples to show the eigenfunctions of the offset FT we obtain. We show the real and the imaginary parts of the first six eigenfunctions $E_{k}(x)$ in Fig. 1 and show their amplitudes $\left|E_{k}(x)\right|$ in Fig. 2. When $\tau$ and $\eta$ are real, the eigenfunctions of the offset FT are the modulation and shifting of the "smoothed" Hermite-Gaussian functions. We will discuss it in Properties D and E in Section III.

Then, we discuss the eigenvalues $\lambda_{k}$ 's of the offset FT. We can also find them from the eigenvalues of the discrete counterpart. From experiments, we find that, if we sort the eigenvectors according to $\left|\tau_{p}\right|$, as in (23), then most of the eigenvalues of the offset DFT can be approximated by the following.

A) When $-N / 4<(p+q) / 2<N / 4$,

$$
\begin{aligned}
& \lambda_{k} \approx j^{N-k} e^{j \pi(p+q)+j \pi(p-q)^{2} /(2 N)} \text { when } k \text { is small } \\
& \lambda_{k} \approx(-j)^{h} e^{j \pi(p+q) / 2+j \pi(p-q)^{2} /(2 N)} \text { when } k \text { is large }
\end{aligned}
$$

B) When $N / 4<(p+q) / 2<3 N / 4$

$$
\begin{aligned}
& \lambda_{k} \approx(-j)^{k} e^{j \pi(p-q)^{2} /(2 N)} \text { when } k \text { is small } \\
& \lambda_{k} \approx(-j)^{h} e^{-j \pi(p+q) / 2+j \pi(p-q)^{2} /(2 N)} \text { when } k \text { is large }
\end{aligned}
$$

In (28) and (29), $h$ is some integer.

To see the eigenvalues, we can apply (25) and set $N$ to be infinite. Then, since

$$
\frac{N}{4}<\frac{p+q}{2}=\sqrt{\frac{N}{2 \pi}} \frac{\tau+\eta}{2}+\frac{N-1}{2}<\frac{3 N}{4}
$$

when $N$ is very large, thus it is proper to apply (29). After substituting (25) into (29), we obtain the following.

\section{- Eigenvalues of the Offset FT:}

$$
\lambda_{k}=(-j)^{k} e^{j \frac{\pi(\tau-\eta)^{2}}{4}} .
$$

The above discussions are valid even when $\tau$ and $\eta$ are complex numbers. Note that, if $\tau$ or $\eta$ is complex, the eigenvalues no longer have unity amplitudes. In this case

$$
\left|\lambda_{k}\right|=\exp [-\pi(r-s)(v-w) / 2]
$$

where $r=\operatorname{real}(\tau), v=\operatorname{imag}(\tau), s=\operatorname{real}(\eta), w=\operatorname{imag}(\eta)$.

We then list several important properties of the eigenfunctions of the offset FT.

(Property A): The complex offset FT has a real eigenfunction set if $\tau=\operatorname{conj}(\eta)$.

Note that any linear operation with a conjugate symmetric kernel has a real eigenfunctions set.

(Property B) Orthogonality Property:

(i) When $\tau$ and $\eta$ are real, and $E_{k}(x)$ and $E_{h}(x)$ are two eigenfunctions of the offset FT, then

$$
\int_{-\infty}^{\infty} E_{k}(x) \overline{E_{h}(x)} d x=C_{k} \delta_{k, h} .
$$


(ii) When $\tau$ and $\eta$ are complex but and $\tau=\eta$, the eigenfunctions are also orthogonal, but now the conjugation operation in (33) is removed

$$
\int_{-\infty}^{\infty} E_{k}(x) E_{h}(x) d x=C_{k} \delta_{k, h} .
$$

Note that in this case the kernel of the offset FT is symmetric.

(iii) If $\tau$ and $\eta$ are complex and $\tau \neq \eta$, eigenfunctions are orthogonal after applying the weighting function $\exp [j(\tau-$ $\eta) x]$

$$
\int_{-\infty}^{\infty} E_{k}(x) E_{h}(x) \exp [j(\tau-\eta) x] d x=C_{k} \delta_{k, h} .
$$

(Property $C$ ): If $E(x)$ is an eigenfunction of the offset FT with parameters $\{\tau, \eta\}$

$$
\mathrm{FT}_{\tau, \eta}[E(x)]=\lambda E(x)
$$

then $\exp (j r x) \cdot E(x)$ will be the eigenfunction of the offset FT with parameter $\{\tau-r, \eta+r\}$ and the eigenvalue is changed into $\exp [j r(\eta+r-\tau)] \cdot \lambda$

$$
\begin{aligned}
\mathrm{FT}_{\tau-r, \eta+r}[\exp (j r x) E(x)]=\exp [ & j r(\eta+r-\tau)] \\
& \times \lambda[\exp (j r x) E(x)] .
\end{aligned}
$$

(Property D) Hermite-Like Property (When $\tau+\eta$ is Real): It is well-known that the original FT has the eigenfunctions of $\exp \left(-x^{2} / 2\right) H_{k}(x)$ where $H_{k}(x)$ is the Hermite function of order $k$. In [12], we found that, for the case of the offset FT with real $\tau$ and $\eta$, the eigenfunctions are the shifting and modulation form of $\exp \left(-x^{2} / 2\right) H_{k}(x)$

$$
E_{k}(x)=e^{j \frac{\eta-\tau}{2} x} \exp \left[-\frac{(2 x-\tau-\eta)^{2}}{8}\right] H_{k}\left(x-\frac{\tau+\eta}{2}\right) .
$$

In fact, (38) can also be applied for the case where $\tau$ and $\eta$ are complex but $\tau+\eta$ is real.

(Property E) Smoothed Hermite-Like Property (When $\tau+\eta$ is Complex): When $\tau+\eta$ is complex, the eigenfunction of the offset FT and the continuous Hermite function may not have the relation as (38). However, we still observe some regularity.

(a) Assume that (i) $E_{k}(x)$ is the $k$ th eigenfunction of the offset FT when $\tau=r+j w$ and $\eta=s+j w$ where $r, s$, and $w$ are real, (ii) $G_{k}(x)$ is the $k$ th eigenfunction of the offset FT when $\tau=r$ and $\eta=s$, and (iii) $|w|$ is small. Then we find that

(1) If $\left|G_{k}(x)\right|$ has a peak (or dip) at $x_{0},\left|E_{k}(x)\right|$ also has a peak (or dip) at $x_{0}$.

(2) $\operatorname{sign}\left[(d / d x)\left|E_{k}(x)\right|\right]=\operatorname{sign}\left[(d / d x)\left|G_{k}(x)\right|\right]$.

(3) $|(d / d x)| E_{k}(x)\left\|<|(d / d x)| G_{k}(x)\right\|$.

In other words, the shape of $\left|E_{k}(x)\right|$ is very similar to that of $\left|G_{k}(x)\right|$, but the variation of $\left|E_{k}(x)\right|$ is smaller. That is, $\left|E_{k}(x)\right|$ is the "smoothed form" of $\left|G_{k}(x)\right|$, and the (a)

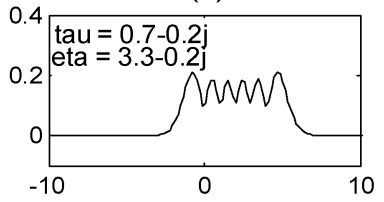

(c)

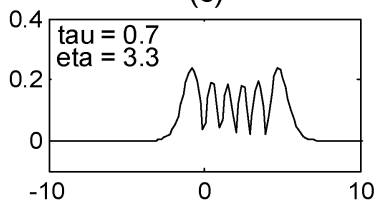

(e)

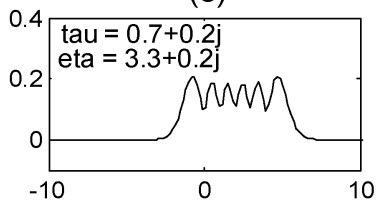

(b)

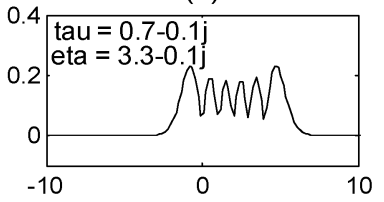

(d)

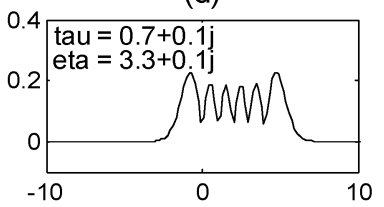

(f)

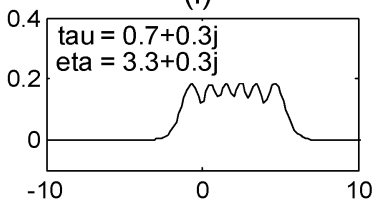

Fig. 3. Comparing the amplitudes of the fifth-order eigenfunctions of the offset FT where $\{\tau, \eta\}=\{0.7+j w, 3.3+j w\}$ and $w=-0.2,-0.1,0,0,0.1$, 0.2 , and 0.3 , respectively. (a) $\left|\mathrm{E}_{5}(\mathrm{x})\right|$. (b) $\left|\mathrm{E}_{5}(\mathrm{x})\right|$. (c) $\left|\mathrm{E}_{5}(\mathrm{x})\right|$. (d) $\mid \mathrm{E}_{5}$ (x)|. (e) $\left|\mathrm{E}_{5}(\mathrm{x})\right|$. (f) $\left|\mathrm{E}_{5}(\mathrm{x})\right|$.

(a)

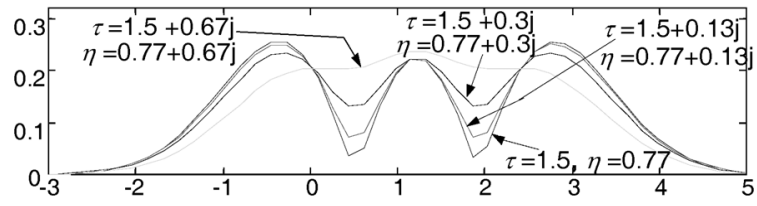

(b)

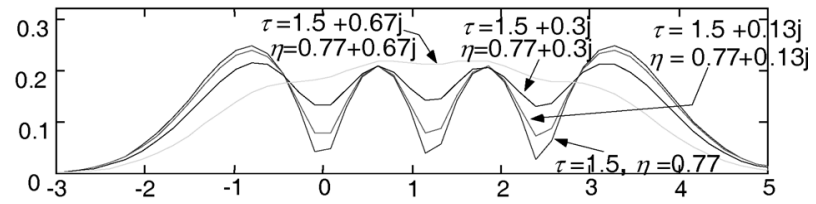

Fig. 4. Comparing the amplitudes of the second- and the third-order eigenfunctions of the offset FT where $\{\tau, \eta\}=\{1.5+j w, 0.77+j w\}$ and $w=0$, $0.13,0.3$, and 0.67 , respectively. (a) $\left|\mathrm{E}_{2}[\mathrm{x}]\right|$. (b) $\left|\mathrm{E}_{3}[\mathrm{x}]\right|$.

eigenfunctions of the offset FT when $\tau+\eta$ is complex is the smoothed Hermite-Gaussian function with shifting and modulation.

In Figs. 3 and 4, we perform some simulations to show how the eigenfunction amplitudes change with $\operatorname{imag}(\tau)$ and $\operatorname{imag}(\eta)$. We find that, as $\operatorname{imag}(\tau)$ and $\operatorname{imag}(\eta)$ grow, the variations of amplitudes are getting smaller and smaller.

(b) Suppose that $E_{k}(x)$ and $F_{k}(x)$ are the $k$ th eigenfunctions of the offset FT with offsets $\{r+j w, s+j v\}$ and $\{r+$ $j(w+z), s+j(v-z)\}$, respectively, then

When $z>0:\left|F_{k}(x)\right|<\left|E_{k}(x)\right|$ for $2 x<\operatorname{Re}(r+s)$

$$
\left|F_{k}(x)\right|>\left|E_{k}(x)\right| \text { for } 2 x>\operatorname{Re}(r+s) .
$$

When $z<0:\left|F_{k}(x)\right|>\left|E_{k}(x)\right|$ for $2 x<\operatorname{Re}(r+s)$

$$
\left|F_{k}(x)\right|<\left|E_{k}(x)\right| \text { for } 2 x>\operatorname{Re}(r+s) \text {. }
$$

These facts can be seen from the experiments in Fig. 5. Note that, if we increase the $\operatorname{imag}(\tau)$ and decrease $\operatorname{imag}(\eta)$, the amplitude of the eigenfunction is "tilted" rightward. 
(a)

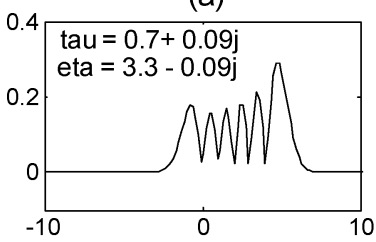

(c)

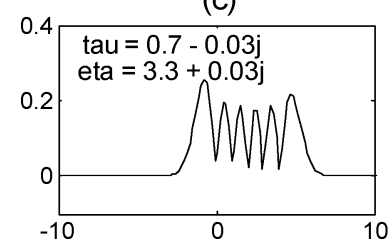

(b)

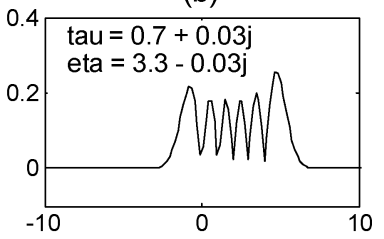

(d)

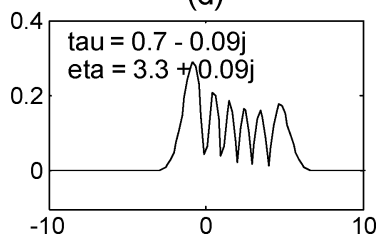

Fig. 5. Comparing the amplitudes of the fifth-order eigenfunctions of the offset FT where $\{\tau, \eta\}=\{0.7-j z, 3.3+j z\}$ and $z=-0.09,-0.03,0.03$, and 0.09 , respectively. (a) $\left|\mathrm{E}_{5}(\mathrm{x})\right|$. (b) $\left|\mathrm{E}_{5}(\mathrm{x})\right|$. (c) $\mid \mathrm{E}_{5}(\mathrm{x}) *$. (d) $\left|\mathrm{E}_{5}(\mathrm{x})\right|$.

(c) If $E_{k}(x)$ and $P_{k}(x)$ are the $k$ th eigenfunctions of the offset FT with offsets $\{\tau, \eta\}$ and $\{\operatorname{conj}(\tau), \operatorname{conj}(\eta)\}$, respectively, then

$$
\left|E_{k}(x)\right|=\left|P_{k}(x)\right|
$$

(Property F) Zero Crossings Property:

(a) When $\tau+\eta$ is real, from (38), since $e^{-j(\eta-\tau) x / 2} E_{k}(x)$ is the shifted version of $\exp \left(-x^{2} / 2\right) H_{k}(x)$ and $H_{k}(x)$ has $k$ zero crossings, thus $e^{-j(\eta-\tau) x / 2} E_{k}(x)$ also has $k$ zero crossings.

(b) When $\tau+\eta$ is complex, from property $\mathrm{E}$, $e^{-j(\eta-\tau) x / 2} E_{k}(x)$ is the "smoothed and shifted version" of $\exp \left(-x^{2} / 2\right) H_{k}(x)$. Thus, although due to smoothing, the number of zero crossings of $e^{-j(\eta-\tau) x / 2} E_{k}(x)$ may not exactly equal to $k$, but it has the trend of growing with $k$.

(c) The numbers of zero crossings of $\operatorname{Re}\left[E_{k}(x)\right]$ and $\operatorname{Im}\left[E_{k}(x)\right]$ depend on those of $e^{-j(\eta-\tau) x / 2} E_{k}(x)$, $\cos (\operatorname{Re}(\eta-\tau) x / 2)$, and $\sin (\operatorname{Re}(\eta-\tau) x / 2)$. Thus, when (1) $k$ is larger and (2) $|\operatorname{Re}(\eta-\tau)|$ is larger, the numbers of zero crossings of $\operatorname{Re}\left[E_{k}(x)\right]$ and $\operatorname{Im}\left[E_{k}(x)\right]$ have the trend of growing larger.

\section{EIGENFUnCTIONS OF FRFTS WITH COMPLEX ORDERS AND OFFSETS}

It is known that, for the nonoffset FRFT with parameter $\alpha$, the eigenfunctions is the Hermite-Gaussian function $\Phi_{k}(x)$ and the corresponding eigenvalue is $\exp (-j \alpha m)$ [2], [3]

$$
O_{F}^{(\alpha, 0,0)}\left[\Phi_{k}(x)\right]=\exp (-j \alpha m) \Phi_{k}(x)
$$

where $\Phi_{k}(x)=\exp \left(-x^{2} / 2\right) H_{k}(x)$ and $H_{k}(x)$ is the $k$ th-order Hermite polynomial. In fact, (43) can also be applied for the case where $\alpha$ is complex. If $\alpha=\phi+j \varphi$ (from (7), $\varphi \leq 0)$, then

$$
O_{F}^{(\phi+j \varphi, 0,0)}\left[\Phi_{k}(x)\right]=\exp (\varphi k) \exp (-j \phi k) \Phi_{k}(x) .
$$

Then, we discuss the case where the offsets $\tau$ and $\eta$ are nonzero. In this case, we can apply (11) and the re-

sult in Section II. Although (11) is nonsense for the case where the amount of displacement is complex, however, we can use it to conclude the relation between the eigenfunction of the offset FT and that of the offset FRFT. Note that the offset FRFT corresponds to the offset LCT where $\{a, b, c, d, \tau, \eta\}=\{\cos \alpha, \sin \alpha,-\sin \alpha, \cos \alpha, \tau, \eta\}$. Thus, from (11), its eigenfunction is

$$
E^{(\alpha, \tau, \eta)}(x)=e^{j\left(\eta-\tau \cot \frac{\alpha}{2}\right) x / 2} E^{(\alpha)}\left(x-\frac{\tau}{2}-\frac{\eta}{2} \cot \frac{\alpha}{2}\right)
$$

where $E^{(\alpha)}(x)$ is the eigenfunction of the nonoffset FRFT. Moreover, since the offset FT corresponds to the case where $\{a, b, c, d, \tau, \eta\}=\{0,1,-1,0, \tau, \eta\}$, thus its eigenfunction is

$$
E_{F}^{(\tau, \eta)}(x)=e^{j(\eta-\tau) x / 2} E_{F}(x-(\tau+\eta) / 2)
$$

where $E_{F}(x)$ is the eigenfunction of the FT. Note that the Gaussian-Hermite function is the eigenfunction for both the FRFT and the FT, i.e., in (43) and (44) we can set $E_{F}(x)=E^{(\alpha)}(x)$. Although (43) and (44) may not be meaningful when $\tau, \eta$, or $\alpha$ is complex, we can conclude that when

$$
\tau+\eta \cot (\alpha / 2)=\tau^{\prime}+\eta^{\prime} \text { and } \eta-\tau \cot (\alpha / 2)=\eta^{\prime}-\tau^{\prime}
$$

i.e.,

$$
\begin{aligned}
\tau^{\prime} & =\frac{\tau}{2}\left(\cot \frac{\alpha}{2}+1\right)+\frac{\eta}{2}\left(\cot \frac{\alpha}{2}-1\right) \\
\eta^{\prime} & =\frac{\tau}{2}\left(1-\cot \frac{\alpha}{2}\right)+\frac{\eta}{2}\left(\cot \frac{\alpha}{2}+1\right)
\end{aligned}
$$

where $\operatorname{Im}(\alpha) \leq 0$, the eigenfunction of the offset FRFT with parameters $\{\alpha, \tau, \eta\}$ is the same as the eigenfunctions of the offset FT with offsets $\left\{\tau^{\prime}, \eta^{\prime}\right\}$ when $\operatorname{Im}(\alpha) \leq 0$. That is the following is true.

\section{- Eigenfunction of the Offset FRFT:}

$$
\begin{aligned}
O_{F}^{(\alpha, \tau, \eta)}[E(x)] & =\lambda^{\alpha}[E(x)] \\
\text { if } \mathrm{FT}_{\tau^{\prime}, \eta^{\prime}}[E(x)] & =\lambda[E(x)]
\end{aligned}
$$

where $\tau^{\prime}$ and $\eta^{\prime}$ are defined in (46). Thus, we can first use the method in Section II to find the eigenfunction of the offset FT with parameters $\left\{\tau^{\prime}, \eta^{\prime}\right\}$, then it is just the eigenfunction of the offset FRFT. To obtain the eigenvalue, we can take fractional power for (31) and obtain that

$$
\lambda_{k}^{\alpha}=(-j)^{\alpha k} e^{j \pi \alpha(\tau \cot (\alpha / 2)-\eta)^{2} / 4+j \kappa}
$$

where $\kappa$ is some real constant. When $\alpha$, or $\eta$ is complex, the amplitude of the eigenvalue may not be 1 .

Since the offset FRFT has the same eigenfunctions as the offset FT, all the properties in Section III can be applied for the offset FRFT with complex parameters and offsets, except that we should change $\{\tau, \eta\}$ as $\left\{\tau^{\prime}, \eta^{\prime}\right\}$ before applying them.

\section{HERMITE-LiKE EIGENFUnCTIONS OF LCTS WiTH COMPLEX PARAMETERS AND OFFSETS}

The LCT with complex parameters and offsets is useful for analyzing the optical system with energy loss [23], [24]. As the works in [11], [12], we will use the following property to derive 
the eigenfunctions of the LCT with complex parameters and offsets.

(Property 1): If (i) $E(x)$ and $\lambda$ are the eigenfunction and the eigenvalue of the operation $O_{A}$, respectively, and (ii) $O_{B}$ is reversible, then since

$$
O_{B} O_{A} O_{B}^{-1}\left\{O_{B}[E(x)]\right\}=O_{B} O_{A}[E(x)]=\lambda \cdot O_{B}[E(x)] .
$$

the operation $O_{B} O_{A} O_{B}^{-1}$ has the eigenfunction of $O_{B}[E(x)]$ and the corresponding eigenvalue is also $\lambda$.

From Property 1, we can derive the eigenfunctions of the offset LCT with complex parameters. In (49), we choose $O_{A}$ as the offset FRFT, which corresponds to the offset LCT with parameters $\left\{\cos \alpha, \sin \alpha,-\sin \alpha, \cos \alpha, \tau_{1}, \eta_{1}\right\}$. Then we choose $O_{B}$ as the offset LCT with parameters $\{e, f, g, h, 0,0\}$, and $O_{B}^{-1}$ is the offset LCT with parameters $\{h,-f,-g, e, 0,0\}$. The constraint is that $f$ should be real, since

$$
\begin{aligned}
& O_{F}^{(h,-f,-g, e, 0,0)}\left\{O_{F}^{(e, f, g, h, 0,0)}[p(x)]\right\} \\
&=\int_{-\infty}^{\infty} \int_{-\infty}^{\infty} \frac{\exp (j(y-x) u / f)}{2 \pi|f|} d u \\
& \quad \times \exp \left[j \frac{e x^{2}-e y^{2}}{2 f}\right] p(x) d x
\end{aligned}
$$

and $(2 \pi|f|)^{-1} \int_{-\infty}^{\infty} \exp \left[j(y-x) f^{-1} u\right] d u=\delta(y-x)$ only when $f$ is real. In (49), if the offset LCT with parameters $\{a, b, c, d, \tau, \eta\}$ equals to $O_{B} O_{A} O_{B}^{-1}$, then

$\left[\begin{array}{ll}a & b \\ c & d\end{array}\right]=\left[\begin{array}{cc}\cos \alpha-(e g+f h) \sin \alpha & \left(e^{2}+f^{2}\right) \sin \alpha \\ -\left(g^{2}+h^{2}\right) \sin \alpha & \cos \alpha+(e g+f h) \sin \alpha\end{array}\right]$

$\left[\begin{array}{l}\tau \\ \eta\end{array}\right]=\left[\begin{array}{ll}e & f \\ g & h\end{array}\right]\left[\begin{array}{l}\tau_{1} \\ \eta_{1}\end{array}\right]$, i.e., $\tau_{1}=\tau h-\eta f, \eta_{1}=-\tau g+\eta e$. where $\operatorname{Im}(a / b) \geq 0$ when $a \neq 0$, and $\operatorname{Im}(b)=0$ when $a=0$ [5]. In (51), since there are 5 unknowns $e, f, g, h$, and $\alpha$ and 4 equalities, there are infinite solutions. To simplify it, we can set

$$
f=1 .
$$

Note that the offset LCT with parameters $\{e, 1, g, h, 0,0\}$ is

$$
(j 2 \pi)^{-0.5} e^{j h u^{2} / 2} \int_{-\infty}^{\infty} \exp (-j u x) \exp \left(j e x^{2} / 2\right) g(x) d x .
$$

It is a combination of the FT and two chirp multiplications. After setting $f=1$, then $e, g, h$, and $\alpha$ can be solved from

$$
\begin{aligned}
a+d & =2 \cos \alpha, \rightarrow \alpha=\cos ^{-1}[(a+d) / 2] \\
b & =\left(e^{2}+1\right) \sin \alpha, \rightarrow e=\sqrt{b \csc \alpha-1} \\
(d-a) \csc \alpha & =2(e g+h), \quad c=-\left(g^{2}+h^{2}\right) \sin \alpha
\end{aligned}
$$

After substituting $g=[(d-a) /(2 e)] \csc \alpha-h / e$ into $c=$ $-\left(g^{2}+h^{2}\right) \sin \alpha$, we obtain

$h=\left[d-a \pm \sqrt{(d+a)^{2}-4} \sqrt{1-b \csc \alpha}\right] /(2 b), g=e h-1$.
Then, all the parameters in (51) can be solved. Note that, in (54), there are two solutions for $e$. To make the integration in (53) finite, it is proper to choose the solution that satisfies imag $(e)>$ 0 . Similarly, in (55), there are also two solutions for $h$ (denoted by $h_{1}$ and $h_{2}$ ). If $\operatorname{imag}\left(h_{1}\right)>0$ and $\operatorname{imag}\left(h_{2}\right)<0$, we set $h=h_{1}$. If both imag $\left(h_{1}\right)$ and imag $\left(h_{2}\right)$ are positive or both of them are negative, we choose the one whose absolute value of the imaginary part is smaller.

Thus, to solve the eigenfunction of the offset LCT with complex parameters and offsets, we can use the process of

(1) First, we use the method in Section IV to solve the eigenfunction of the offset FRFT with parameter $\left\{\alpha, \tau_{1}, \eta_{1}\right\}$ where $\alpha, \tau_{1}$, and $\eta_{1}$ are defined in (54) and (51).

$$
O_{F}^{\left(\alpha, \tau_{1}, \eta_{1}\right)}\left[E_{1}(x)\right]=\lambda E_{1}(x) .
$$

(2) Then we do the following operation, which is equivalent to the offset LCT with parameters $\{e, 1, g, h, 0,0\}$, for $E_{1}(x)$. The result $E(x)$ is the eigenfunction of the offset LCT and the eigenvalue is also $\lambda$.

$$
E(x)=\exp \left(j h x^{2} / 2\right) \mathrm{FT}\left[\exp \left(j e v^{2} / 2\right) E_{1}(v)\right]
$$

where $e$ and $h$ are derived from (54) and (55)

$$
O_{F}^{(a, b, c, d, \tau, \eta)}[E(x)]=\lambda E(x), \lambda \text { is defined in (56) . }
$$

\section{OTHER TYPES OF EIGENFUNCTION}

\section{A. Periodic Function-Like Eigenfunctions}

It is known that the eigenfunction of the Fresnel transform is a periodic function. Therefore, in (49), we can also choose $O_{A}$ as the Fresnel transform (i.e., the LCT with parameters $\{1, r, 0,1\})$. Then we also choose $O_{B}$ as the offset LCT with parameters $\left\{a_{1}, b_{1}, c_{1}, d_{1}\right\}$. Then, we obtain that

$$
\begin{aligned}
{\left[\begin{array}{ll}
a & b \\
c & d
\end{array}\right] } & =\left[\begin{array}{ll}
a_{1} & b_{1} \\
c_{1} & d_{1}
\end{array}\right]\left[\begin{array}{cc}
1 & r \\
0 & 1
\end{array}\right]\left[\begin{array}{cc}
d_{1} & -b_{1} \\
-c_{1} & a_{1}
\end{array}\right] \\
& =\left[\begin{array}{cc}
1-a_{1} c_{1} r & a_{1}^{2} r \\
-c_{1}^{2} r & 1+a_{1} c_{1} r
\end{array}\right] .
\end{aligned}
$$

There are infinite solutions for $a_{1}, c_{1}$, and $r$. Since the eigenvectors of the Fresnel transform is a periodic function

$$
O_{F}^{(1, r, 0,1)}(f(t))=\mu f(t) \text { if } f(t)=\mu f(t+\sqrt{|r| \pi})
$$

thus, from (49), the offset LCT has the eigenfunction of the following form, which is the LCT of a periodic function, and the corresponding eigenvalue is $\mu$

$$
E(x)=e^{j d_{1} x^{2} /\left(2 b_{1}\right)} \int_{-\infty}^{\infty} e^{-j x y / b_{1}} e^{j a_{1} y^{2} /\left(2 b_{1}\right)} f(y) d y
$$

where $a_{1}, b_{1}, c_{1}, d_{1}$, and $r$ should satisfy (59) and $f(x)$ should satisfy (60). 


\section{B. Fractal-Like Eigenfunctions}

Similarly, if in (49), we choose $O_{A}$ as a scaling operation, i.e., the offset LCT with parameter $\left\{\sigma^{-1}, 0,0, \sigma, 0,0\right\}$, then

$$
\begin{aligned}
{\left[\begin{array}{ll}
a & b \\
c & d
\end{array}\right] } & =\left[\begin{array}{ll}
a_{1} & b_{1} \\
c_{1} & d_{1}
\end{array}\right]\left[\begin{array}{cc}
\sigma^{-1} & 0 \\
0 & \sigma
\end{array}\right]\left[\begin{array}{cc}
d_{1} & -b_{1} \\
-c_{1} & a_{1}
\end{array}\right] \\
& =\left[\begin{array}{cc}
a_{1} d_{1} \sigma^{-1}-b_{1} c_{1} \sigma & a_{1} b_{1}\left(\sigma-\sigma^{-1}\right) \\
c_{1} d_{1}\left(\sigma^{-1}-\sigma\right) & -b_{1} c_{1} \sigma^{-1}+a_{1} d_{1} \sigma
\end{array}\right] \\
\text { where } \sigma & =\left[a+d \pm \sqrt{(a+d)^{2}-4}\right] / 2 .
\end{aligned}
$$

Since the eigenfunction of the scaling operation is the scalinginvariant function (i.e., the fractal)

$$
s(x)=\lambda s(\sigma x)
$$

thus the offset LCT has the following eigenfunction, which is the LCT of a fractal, and the corresponding eigenvalue is also $\lambda\left(\left\{a_{1}, b_{1}, c_{1}, d_{1}\right\}, \sigma\right.$, and $s(x)$, should satisfy (62), (63), and (64))

$$
E(x)=e^{j d_{1} y^{2} /\left(2 b_{1}\right)} \int_{-\infty}^{\infty} e^{-j x y / b_{1}} e^{j a_{1} y^{2} /\left(2 b_{1}\right)} s(y) d y .
$$

\section{EIGENFUNCTIONS/EIGENVECTORS of Continuous/Discrete Cosine, Sine, AND HARTLEY TRANSFORMS}

Since the cosine transform (CT), sine, transform (ST), and Hartley transform (HT) have close relation with the FT (even with the complex offset terms), we can use the eigenfunction of the complex offset FT to derive the eigenfunctions of the CT, ST, and HT with complex offset. Their definitions are offset CT, ST, and HT are defined as follows.

\section{- Offset CT:}

$$
\mathrm{CT}_{\tau, \eta}[g(x)]=\sqrt{1 / 2 \pi} \int_{-\infty}^{\infty} \cos [(x-\eta)(\omega-\tau)] g(x) d x \text {. }
$$

\section{- Offset ST:}

$$
\mathrm{ST}_{\tau, \eta}[g(x)]=\sqrt{1 / 2 \pi} \int_{-\infty}^{\infty} \sin [(x-\eta)(\omega-\tau)] g(x) d x
$$

\section{- Offset HT:}

$$
\operatorname{HT}_{\tau, \eta}[g(x)]=\sqrt{1 / 2 \pi} \int_{-\infty}^{\infty} \operatorname{cas}[(x-\eta)(\omega-\tau)] g(x) d x
$$

In the above, $\operatorname{cas}(x)=\cos (x)+\sin (x)$. We find that

(A) The eigenfunctions of the FT with offsets $(\tau, \tau)$ (i.e., $\eta=\tau)$ are also the eigenfunctions of the CT with offsets $(\tau, \tau)$. It can be proved from the following fact.

(i) The offset FT with parameters $(\tau, \eta)$ and the offset IFT with parameters $(\tau, \eta)$ (defined in (1) and (2)) have the same eigenfunctions. That is,

$$
\text { if } \mathrm{FT}_{\tau, \eta}\left[E_{k}(x)\right]=\lambda_{k} E_{k}(x)
$$

$$
\text { then } \operatorname{IFT}_{\tau, \eta}\left[E_{k}(x)\right]=\lambda_{k}^{-1} E_{k}(x) .
$$

(ii) When $\tau=\eta$, then

$$
\begin{aligned}
& \mathrm{FT}_{\tau, \eta}[g(x)]+\mathrm{IFT}_{\tau, \eta}[g(x)] \\
& \quad=\sqrt{1 / 2 \pi} \int_{-\infty}^{\infty}\left(e^{-j(\omega-\tau)(x-\tau)}+e^{j(\omega-\tau)(x-\tau)}\right) g(x) d x \\
& \quad=2 C T_{\tau, \eta}(g(x))
\end{aligned}
$$

(iii) Therefore, if $E_{k}(x)$ is an eigenfunction of the offset FT with parameters $(\tau, \tau)$, then

$$
\begin{aligned}
\mathrm{CT}_{\tau, \tau}\left[E_{k}(x)\right] & =\left\{\mathrm{FT}_{\tau, \tau}\left[E_{k}(x)\right]+I \mathrm{FT}\right. \\
& =\left(\lambda_{k}+\lambda_{k}^{-1}\right) E_{k}(x) / 2 .
\end{aligned}
$$

That is, $E_{k}(t)$ is also the eigenfunction of the offset CT with parameters $(\tau, \tau)$ and the corresponding eigenvalue is $\left(\lambda_{k}+\lambda_{k}^{-1}\right) / 2$.

(B) If $E_{k}(x)$ is an eigenfunction of the offset FT with parameters $(\tau, \tau)$ and the eigenvalue is $\lambda_{k}$, then $E_{k}(x)$ is also an eigenfunction of the offset Sine and HTs, and the eigenvalues are

$$
\begin{aligned}
& j\left(\lambda_{k}-\lambda_{k}^{-1}\right) / 2, \quad \text { for offset ST } \\
& (1+j) \lambda_{k} / 2+(1-j) \lambda_{k}^{-1} / 2, \quad \text { for offset HT. }
\end{aligned}
$$

The proofs are similar to that of the offset CT.

(C) When $\tau \neq \eta$, there is no obvious relation between the eigenfunction of the offset FT and those of the offset CT, ST, and HTs.

(D) Similarly, for the offset discrete CT, ST, and HTs (offset DCT, DST, DHT)

$$
\begin{aligned}
\operatorname{DCT}_{p, q}(x[n])= & \sqrt{N^{-1}} \sum_{n=0}^{N-1} \cos \left[\frac{2 \pi(m-p)(n-q)}{N}\right] \\
& \times x[n] \\
\operatorname{DST}_{p, q}(x[n])= & \sqrt{N^{-1}} \sum_{n=0}^{N-1} \sin \left[\frac{2 \pi(m-p)(n-q)}{N}\right] \\
& \times x[n] \\
\operatorname{DHT}_{p, q}(x[n])= & \sqrt{N^{-1}} \sum_{n=0}^{N-1} \operatorname{cas}\left[\frac{2 \pi(m-p)(n-q)}{N}\right] \\
& \times x[n]
\end{aligned}
$$

we find that, if $v_{k}[n]$ is an eigenvector of the offset DFT with parameters $(p, p)$ and the eigenvalue is $\mu_{k}$, then $v_{k}[n]$ is an eigenvector of the offset DCT, DST, and DHT with parameters $(p, p)$, and the corresponding eigenvectors are

$$
\begin{aligned}
& \text { for offset DCT }:\left(\mu_{k}+\mu_{k}^{-1}\right) / 2 \\
& \text { for offset DST }: j\left(\lambda_{k}-\lambda_{k}^{-1}\right) / 2 \\
& \text { for offset DHT }:\left[(1+j) \lambda_{k}+(1-j) \lambda_{k}^{-1}\right] / 2 \text {. }
\end{aligned}
$$

When $p \neq q$, there is no obvious relation between the eigenvectors of the offset DFT and those of the offset DCT, DST, and DHT. 


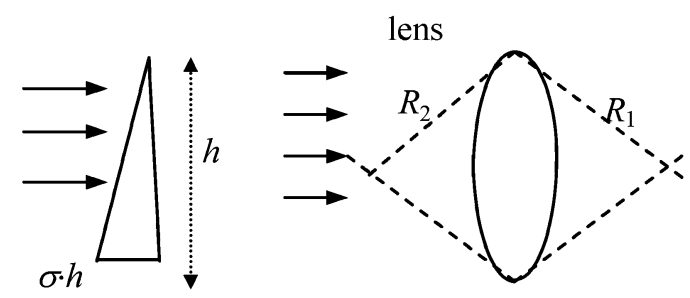

Fig. 6. Lens and a prism in an optical system may absorb the light energy.

\section{APPLICATIONS}

\section{A. Self-Imaging Phenomenon Analysis}

In many cases, especially when a part of light energy is lost after the light propagating through some components of an optical system, we should use the offset LCT with complex variables to analyze the optical system [23], [24].

For example, the prism in Fig. 6 has the transfer function as

$$
h_{o}(x)=t(x) h_{i}(x)
$$

where

$$
\begin{gathered}
t(x)=\exp [-j k(n-1) \sigma x], \quad k=2 \pi \lambda^{-1} \\
\sigma=\frac{\text { bottom width of the prism }}{\text { height of the prism }} \\
n: \text { refractive index. }
\end{gathered}
$$

Since (80) is a modulation operation, we can use the offset LCT with the parameters of

$$
\{a, b, c, d, \tau, \eta\}=\{1,0,0,1,0,-k(n-1) \sigma\}
$$

to represent it. Note that all the parameters in (81) are real. However, if the lens can absorb the energy of light, the transfer function becomes

$$
t(x)=\exp [-\mu(h-x)] \exp [-j k(n-1) \sigma x]
$$

where $h$ is the height of the prism. In this case, using the offset LCT with real parameters to analyze it will not be suitable and we should the offset LCT with complex variables to represent it. Comparing with (5), it can be represented by the offset LCT with parameters

$$
\begin{aligned}
\{a, b, c, d, \tau, \eta\} & =\left\{1,0,0,1,0,-\mu_{1}-j \mu_{2}\right\} \\
\mu_{1} & =k(n-1) \sigma, \quad \mu_{2}=\mu .
\end{aligned}
$$

Another example is that the transfer function for a lens in Fig. 6 can be expressed as (suppose that it can absorb the energy

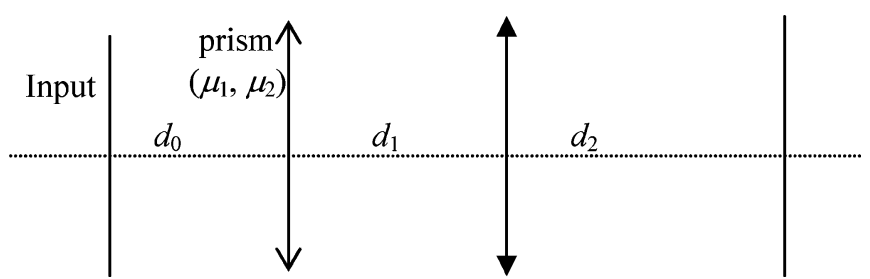

Fig. 7. Optical system consisted of a decayed component.

of the light)

$$
\begin{aligned}
h_{o}(x) & =t(x) h_{i}(x), \text { where } \\
t(x) & =\exp \left[-j k x^{2} /(2 f)\right] \exp \left[-\rho\left(h^{2}-x^{2}\right) /(2 f)\right]
\end{aligned}
$$

and $f$ is the focal length of the lens. Comparing with (5), we can conclude that it can be represented by the offset LCT with

$$
\begin{aligned}
\{a, b, c, d, \tau, \eta\} & =\left\{1,0,-\rho_{1}-j \cdot \rho_{2}, 1,0,0\right\} \\
\rho_{1} & =k / f, \quad \rho_{2}=\rho / f .
\end{aligned}
$$

If an optical system consists of the light-absorption components as in (82) or (84), we must use the offset LCT with complex parameters to represent it.

Then, we see the optical system in Fig. 7. It consists of a lens, a prism, and three free spaces. We can apply (83), (85), and the additivity property of the offset LCT in (9) and (10). After some computation, we can show that the system in Fig. 7 can be expressed as the offset LCT with parameters shown in (86) and (87) at the bottom of the page. All the six parameters may have imaginary part.

In [12], we derived the eigenfunctions of the offset FT and the offset LCT with real parameters and offsets, see (11). However, (11) can not be applied for the case where the parameters and offsets are complex. Now, since we have derived the eigenfunctions of the LCT with complex parameters and offsets, we can use them to analyze the self-imaging phenomenon and the resonance phenomenon for the optical system with decayed components.

For the example in Fig. 7, if we choose the parameter as

$$
\begin{array}{ll}
d_{0}=1, & d_{1}=1.45, \quad d_{2}=2, \quad k=10 \\
\rho_{1}=2, & \rho_{2}=0.5, \quad \mu_{1}=1, \quad \mu_{2}=0.2
\end{array}
$$

then it can be represented by the offset LCT with parameters

$$
\begin{aligned}
{\left[\begin{array}{ll}
a & b \\
c & d
\end{array}\right] } & =\left[\begin{array}{cc}
0.6-0.1 j & 0.347-0.0245 j \\
-2-0.5 j & 0.51-0.1225 j
\end{array}\right] \\
{\left[\begin{array}{l}
\tau \\
\eta
\end{array}\right] } & =\left[\begin{array}{ll}
-0.2899-0.0429 j \\
-0.7245-0.0695 j
\end{array}\right] .
\end{aligned}
$$

$$
\begin{aligned}
{\left[\begin{array}{ll}
a & b \\
c & d
\end{array}\right] } & =\left[\begin{array}{cc}
1-\frac{d_{2} \rho_{1}}{k}-j \frac{d_{2} \rho_{2}}{k} & \frac{d_{0}+d_{1}+d_{2}}{k}-\frac{\left(d_{0}+d_{1}\right) d_{2} \rho_{1}}{k^{2}}-j \frac{\left(d_{0}+d_{1}\right) d_{2} \rho_{2}}{k^{2}} \\
-\rho_{1}-j \rho_{2} & 1-\left(d_{0}+d_{1}\right) \rho_{1} k^{-1}-j\left(d_{0}+d_{1}\right) \rho_{2} k^{-1}
\end{array}\right] \\
\tau & =d_{1} d_{2} k^{-2}\left(\rho_{1} \mu_{1}-\rho_{2} \mu_{2}\right)-\left(d_{1}+d_{2}\right) \mu_{1} k^{-1}+j\left[d_{1} d_{2} k^{-2}\left(\rho_{2} \mu_{1}+\rho_{1} \mu_{2}\right)-\left(d_{1}+d_{2}\right) \mu_{2} k^{-1}\right] \\
\eta & =\left(d_{1} k^{-1} \rho_{1}-1\right) \mu_{1}-d_{1} k^{-1} \rho_{2} \mu_{2}+j\left[\left(d_{1} k^{-1} \rho_{1}-1\right) \mu_{2}+d_{1} k^{-1} \rho_{2} \mu_{1}\right] .
\end{aligned}
$$


(a)

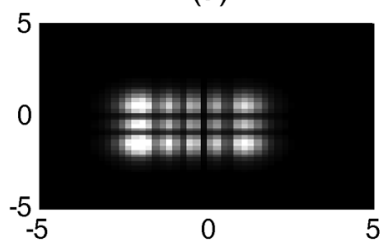

(c)

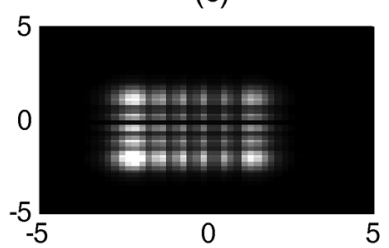

(b)

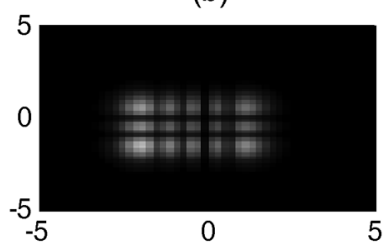

(d)

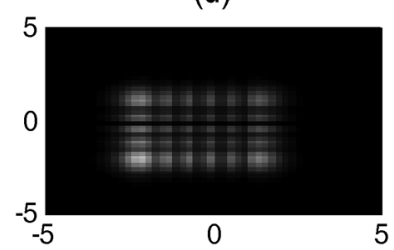

Fig. 8. (a) Eigenmode of $m_{x}=2$ and $m_{y}=4\left(m_{x}\right.$ and $m_{y}$ are the orders of the eigenfunction in $x$ axis and $y$-axis, respectively) for the optical system in Fig. 7. (b) Output when the input is (a). (c) Eigenmode of $m_{x}=4$ and $m_{y}=5$, (d) Output when the input is (c). (a) $E_{2,4}(x, y)$. (b) Output for $E_{2,4}(x, y)$. (c) $\mathrm{E}_{4,5}(\mathrm{x}, \mathrm{y})$. (d) Output for $\mathrm{E}_{4,5}(\mathrm{x}, \mathrm{y})$.

We apply the results in Section V and obtain its eigenfunction. In Fig. 8, we show an example of applying the modes of $\left\{k_{x}=\right.$ $\left.2, k_{y}=4\right\}$ and $\left\{k_{x}=4, k_{y}=5\right\}$ to the optical system in Fig. 7 and show the input and the output, where $k_{x}$ and $k_{y}$ mean the orders of the eigenfunctions for $x$ axis and $y$-axis, respectively. Observing the outputs, we find that some energy is lost after the light propagating through the optical system. It is due to that the eigenvalues of the offset FT, FRFT, and LCT with complex parameters and offsets may not have unitary amplitudes.

\section{B. Fractional Laplace and Fractional Z-transforms}

Note that, from (38), for the offset FT with parameters

$$
(\tau, \eta)=(j \sigma,-j \sigma)
$$

the eigenfunctions are the decayed forms of Hermite-Gaussian functions $\Phi_{k}(x)$

$$
E_{k}^{(j \sigma, j \sigma)}(x)=e^{\sigma x} \Phi_{k}(x), \text { where } \Phi_{k}(x)=e^{-x^{2} / 2} H_{k}(x)
$$

and the corresponding eigenvectors are $(-j)^{k} \exp \left(-j \sigma^{2}\right)$. The fractional Laplace transform can be defined as the fractional operation of the offset FT with parameter $(j \sigma,-j \sigma)$

From [3], the nonoffset FRFT is derived from eigenfunction summation

$$
\begin{aligned}
G_{\alpha}(u) & =\int_{-\infty}^{\infty} g(x) \sum_{k=0}^{\infty} e^{-j \alpha k} \\
& \times \tilde{\Phi}_{k}(x) \Phi_{k}(u) d x \\
\text { where } \tilde{\Phi}_{k}(x) & =\Phi_{k}(x) / \int_{-\infty}^{\infty} \Phi_{k}^{2}(x) d x \\
\int_{-\infty}^{\infty} \Phi_{j}(x) \tilde{\Phi}_{k}(x) d x & =1, \quad \text { when } j=k \\
\int_{-\infty}^{\infty} \Phi_{j}(x) \tilde{\Phi}_{k}(x) d x & =0, \quad \text { otherwise. }
\end{aligned}
$$


the discrete FRFT (DFRFT) without offset and $\mathbf{Z}_{\alpha}$ is the transform matrix of the fractional $Z$-transform, then

$$
Z_{\alpha}[m, n]=e^{j \frac{2 \pi}{N} \sigma^{2}} e^{\frac{2 \pi}{N} \sigma(m-n)} F_{\alpha}[m, n]
$$

Therefore, the fractional $Z$-transform is defined as

$$
G_{Z, \alpha}[m]=\sum_{n=0}^{N-1} Z_{\alpha}[m, n] g[n]
$$

where $Z_{\alpha}[m, n]$ has the relation in (101) with the transform matrix of the DFRFT. We can also prove that the fractional $Z$-transform is additive and reversible

$$
\mathbf{Z}_{\alpha} \mathbf{Z}_{\beta}=\mathbf{Z}_{\alpha+\beta}, \quad \mathbf{Z}_{-\alpha} \mathbf{Z}_{\alpha}=\mathbf{I} \quad(\mathbf{I} \text { is an identity matrix }) .
$$

When $\alpha=\pi / 2$, the fractional $Z$-transform become the original $Z$ transform

$$
\begin{gathered}
G_{Z, \alpha}[m, n]=e^{-j 2 \pi \sigma^{2} / N} e^{2 \pi \sigma m / N} G(z) \\
\text { where } G(z) \text { is the original Z-transform of } g[n] \\
\text { and } z=\exp (j 2 \pi m / N) \exp (2 \pi \sigma / N) .
\end{gathered}
$$

Both the fractional Laplace transform and the fractional $Z$-transform are additive. They will be useful for signal processing. As the FRFT and the DFRFT, they are suitable for processing the signal whose instantaneous spectrum varies with time. Moreover, for the following signal

$$
\begin{aligned}
& \int_{-\infty}^{\infty}|g(x)| d x \text { or } \sum_{n=-\infty}^{\infty}|g[n]| \text { does not converge } \\
& \text { but } \int_{-\infty}^{\infty} e^{-\sigma x}|g(x)| d x \text { or } \\
& \sum_{n=-\infty}^{\infty} e^{-\sigma n}|g[n]| \text { converges for some } \sigma .
\end{aligned}
$$

Although they cannot be transformed by the original FRFT and the DFRFT, they can be processed by the fractional Laplace and $Z$-transforms.

\section{Other Applications}

(i) Analyzing the resonance phenomena:

We can use the derived eigenfunctions to analyze the resonance phenomena for the radar system, the gradient fiber system [21], or other electromagnetic wave systems with energy absorbing components: The method is similar to that for self-imaging phenomena analysis.

(ii) Mode selection:

Even when the offsets $\tau^{\prime}$ and $\eta^{\prime}$ are complex, the eigenfunctions of the offset FT form a complete set. That is, any function $f(x)$ can be expanded as a summation of the eigenfunction of the offset FT, as in (106), where $E_{k}(x)$ is the eigenfunction of the offset FT

$$
f(x)=\sum_{k=0}^{\infty} a_{k} E_{k}(x)
$$

Moreover, from (45) and (46) in Section IV, we know that the offset FRFT with parameters $\{\alpha, \eta, \tau\}$ and the offset FT with offsets $\left\{\tau^{\prime}, \eta^{\prime}\right\}$ have the same eigenfunctions and their eigenvalues have the fractional power relation, see (45)-(48). Thus, analogous to the works in [22], if $a_{r} \approx 0$ for $r>M$

$$
\begin{aligned}
\tau & =\sin \psi_{m}\left[\tau^{\prime}\left(\sin \psi_{m}+\cos \psi_{m}\right)+\eta^{\prime}\left(\sin \psi_{m}-\cos \psi_{m}\right)\right] \\
\psi_{m} & =\pi m M^{-1} \\
\eta & =\sin \psi_{m}\left[\tau^{\prime}\left(\cos \psi_{m}-\sin \psi_{m}\right)\right. \\
& \left.\quad+\eta^{\prime}\left(\cos \psi_{m}+\sin \psi_{m}\right)\right]
\end{aligned}
$$

we can obtain a certain mode $a_{k} E_{k}(x)$. It is useful for phase retrieval and spectrum analysis.

(iii) Generalization for the DFRFT

Note that, in Section II we derived not only the eigenfunctions of the offset FT but also the eigenvectors of its discrete counterpart, the offset DFT. We can apply these eigenvectors to define a general form of the fractional offset DFT i.e.,

$$
\mathbf{F}_{\mathbf{p}, \mathbf{q}}^{\alpha}=\mathbf{V}_{\mathbf{p}, \mathbf{q}} \mathbf{D}_{\mathbf{p}, \mathbf{q}}^{\alpha} \mathbf{V}_{\mathbf{p}, \mathbf{q}}^{-1}
$$

where

$$
\begin{aligned}
& \mathbf{V}_{\mathbf{p}, \mathbf{q}}=\left[\begin{array}{lllll}
\mathbf{v}_{\mathbf{0}}^{\mathbf{T}} & \mathbf{v}_{\mathbf{1}}^{\mathbf{T}} & \cdots & \cdots & \mathbf{v}_{\mathbf{N}-\mathbf{1}}^{\mathbf{T}}
\end{array}\right] \\
& \mathbf{D}_{\mathbf{p}, \mathbf{q}}^{\alpha} \quad \text { is diagonal, } \quad \mathbf{D}_{\mathbf{a}, \mathbf{b}}^{\alpha}[n]=\exp \left(j 2 \pi \alpha M_{n}\right) \lambda_{n}^{\alpha} \\
& M_{n}{ }^{\prime} \quad \mathrm{s} \text { are any integers, } \\
& \mathbf{v}_{\mathbf{k}} \text { 's : the normalized eigenvectors ofthe offset } \\
& \text { DFT } \mathbf{F}_{\mathbf{p}, \mathbf{q}} \text { derived in Section II-B } \\
& \lambda_{k} \text { 's : the corresponding eigenvalues } \\
& \text { see (28) and (29). }
\end{aligned}
$$

The fractional offset DFT are much more flexible and general than the original DFT and the existing fractional DFT [18], [20], and all of their applications are also the potential applications of the fractional offset DFT. We can use the advantage that the fractional offset DFT has a lot of adjustable parameters ( $p, q, \alpha$, and $M_{n}$ 's) to tune for better performance. It will be useful for encryption, filter design, watermark, pattern recognition, and linear system modeling.

\section{Summary AND CONCLUSION}

The eigenvectors and eigenfunctions of the offset and nonoffset DFT, FT, FRFT, and LCT are summarized in Tables I and II. In this paper, we derived the eigenfunctions of the offset FT with arbitrary offsets $\tau$ and $\eta$, including the case where $\tau, \eta$, or both of them are complex numbers (i.e., the decayed offset FT). We found the eigenfunctions of the offset FT with complex $\tau$ and $\eta$ are the smoothed Hermite-Gaussian functions with shifting and modulation. We also extended the results for the 
TABLE I

SUMMARY OF THE EIGENVECTORS OF THE NONOFFSET AND OFFSET DFT

\begin{tabular}{|l|l|}
\hline \multicolumn{1}{|c|}{ Operations } & \multicolumn{1}{|c|}{ Types of Eigenvectors and References } \\
\hline (1) DFT & Hermite-Gaussian like vectors, derived from S matrix (Ref. [13]) \\
\hline $\begin{array}{l}\text { (2) Offset DFT for } p=q \\
\text { 1/2 }\end{array}$ & Shifted version of Hermite-Gaussian-like vectors, Ref. [14] \\
\hline $\begin{array}{l}\text { (3) Offset DFT for } p+q \\
\text { any integer }\end{array}$ & $\begin{array}{l}\text { (i) Shifted and (ii) modulation version of Hermite-Gaussian } \\
\text { like vectors, Ref. [15] }\end{array}$ \\
\hline $\begin{array}{l}\text { (4) Offset DFT for } p=q \\
\text { arbitrary real } p \text { and } q,\end{array}$ & $\begin{array}{l}\text { Shifted version of Hermite-Gaussian like vectors, Refs. [16][17] } \\
\text { like vectors, derived from the commuting matrix in Eq. (18) of } \\
\text { this paper }\end{array}$ \\
\hline $\begin{array}{l}\text { (6) Offset DFT for } \\
\text { arbitrary } p \text { and } q,\end{array}$ & $\begin{array}{l}\text { (i) Smoothed, (ii) shifted, and (iii) modulation version of } \\
\text { Hermite-Gaussian-like vectors, derived from the commuting } \\
\text { matrix in Eq. (18) of this paper }\end{array}$ \\
\hline $\begin{array}{l}\text { (7) Offset DCT, DST, } \\
\text { and DHT for } p=q\end{array}$ & $\begin{array}{l}\text { Eigenvectors are the same as those of the DFT with offsets ( } p, \\
p \text { ), see Section VII in this paper }\end{array}$ \\
\hline
\end{tabular}

TABLE II

SUMMARY OF THE EIGENFUNCTIONS OF THE CONTINUOUS NONOFFSET AND OFFSET FT, FRFT, AND LCT

\begin{tabular}{|c|c|}
\hline Operations & Types of Eigenvectors and References \\
\hline (1) FT, FR & $x^{2} / 2 H_{k}(x)$, Hermite-Gaussian functions (Refs. [1][2][3]) \\
\hline (2) LCT & $\begin{array}{l}\text { (a) Hermite-Gaussian functions + scaling + chirp multiplication } \\
\text { (b) Periodic Functions with chirp multiplication and FT } \\
\text { (c) Fractals with chirp multiplication and chirp convolution } \\
\text { (Eqs. (39), (56), (62), (98), (109), (132), and (146) in Ref. [11]) }\end{array}$ \\
\hline $\begin{array}{l}\text { (3) Offset FT and } \\
\text { FRFT }(\eta+\tau \text { is real })\end{array}$ & $\begin{array}{l}\quad E_{\tau, \eta}(t)=e^{j(\eta-\tau) x / 2} e^{-[x-(\tau+\eta) / 2]^{2} / 2} H_{k}(x-(\tau+\eta) / 2) \text {, } \\
\text { Hermite-Gaussian functions with (i) modulation and (ii) } \\
\text { shifting, Eq. (47) in Ref. [12]. }\end{array}$ \\
\hline $\begin{array}{l}\text { (4) Offset LCT } \\
(\eta+\tau \text { is real) }\end{array}$ & $\begin{array}{l}\text { (i) Modulation and (ii) shifting for the three type eigenfunctions } \\
\text { of the LCT, Eq. (60) in Ref. [12] }\end{array}$ \\
\hline $\begin{array}{l}\text { (5) Offset FT / FRFT } \\
(\eta+\tau \text { is complex })\end{array}$ & $\begin{array}{l}\text { Hermite-Gaussian functions with (i) modulation, (ii) shifting, } \\
\text { and (iii) Smoothing (illustrated in property E of Section III), } \\
\text { derived from the commuting matrix in (18) together with Eqs. } \\
\text { (46) and (47) in this paper. }\end{array}$ \\
\hline $\begin{array}{l}\text { (6) Offset LCT } \\
(\eta+\tau \text { is complex })\end{array}$ & 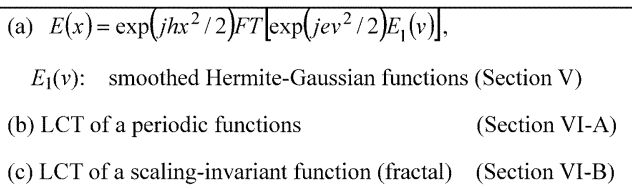 \\
\hline $\begin{array}{l}\text { (7) Offset Cosine, } \\
\text { Sine, and Hartley } \\
\text { transforms when } \tau=\eta\end{array}$ & $\begin{array}{l}\text { Eigenfunctions are same as those of the FT with offsets }(\tau, \tau) \text {. See } \\
\text { Section VII in this paper. }\end{array}$ \\
\hline
\end{tabular}

case of the offset FRFT and the offset LCT with complex parameters and offsets. The eigenfunctions we obtained are useful for analyzing the optical system consisting of energy-absorbing components, resonance analysis, mode selection, and defining the fractional Laplace transform, fractional $Z$-transform, or a much more general form of the DFRFT.

\section{REFERENCES}

[1] R. N. Bracewell, The Fourier Integral and Its Applications. Boston, MA: McGraw Hill, 2000.

[2] H. M. Ozaktas, M. A. Kutay, and Z. Zalevsky, The Fractional Fourier Transform With Applications in Optics and Signal Processing. New York: Wiley, 2000.

[3] V. Namias, "The fractional-order Fourier transform and its application to quantum mechanics," J. Inst. Maths. Appl., vol. 25, pp. 241-265, 1980.

[4] L. B. Almeida, "The fractional Fourier transform and time-frequency representations," IEEE Trans. Signal Process., vol. 42, no. 11, pp. 3084-3091, Nov. 1994.

[5] K. B. Wolf, "Ch. 9: Canonical transforms," in Integral Transforms in Science and Engineering, K. B. Wolf, Ed. New York: Plenum, 1979, pp. 381-416.

[6] G. Bongiovanni, P. Corsini, and G. Frosini, "One-dimensional and two-dimensional generalized discrete Fourier transforms," IEEE Trans. Acoust., Speech, Signal Process., vol. ASSP-24, no. 1, pp. 97-99, Feb. 1976.

[7] O. K. Erosy, "Semisystolic array implementation of circular, skew-circular, and linear convolutions," IEEE Trans. Comput., vol. COM-34, no. 2, pp. 190-194, Feb. 1985.

[8] S. Abe and J. T. Sheridan, "Optical operations on wave functions as the Abelian subgroups of the special affine Fourier transformation," Opt. Lett., vol. 19, pp. 1801-1803, 1994.

[9] S. Abe and J. T. Sheridan, "Generalization of the fractional Fourier transformation to an arbitrary linear loseless transformation: An operator approach," J. Phys. A, vol. 27, pp. 4179-4187, 1994.

[10] D. F. V. James and G. S. Agarwal, "The generalized Fresnel transform and its applications to optics," Opt. Commun., vol. 126, pp. 207-212, 1996.

[11] S. C. Pei and J. J. Ding, "Eigenfunctions of linear canonical transform," IEEE Trans. Signal Process., vol. 50, no. 1, pp. 11-26, Jan. 2002.

[12] S. C. Pei and J. J. Ding, "Eigenfunctions of the offset Fourier, fractional Fourier, and linear canonical transforms," J. Opt. Soc. Am. A, vol. 20, pp. 522-532, 2003.

[13] B. W. Dickinson and K. Steiglitz, "Eigenvectors and functions of the discrete Fourier transform," IEEE Trans. Acoust, Speech, Signal Process., vol. ASSP-30, no. 1, pp. 25-31, Jan. 1982.

[14] C. C. Tseng, "Eigenvalues and eigenvectors of generalized DFT, generalized DHT, DCT-IV and DST-IV matrices," IEEE Trans. Signal Process., vol. 50, no. 4, pp. 866-877, Apr. 2002.

[15] S. C. Pei and J. J. Ding, "Generalized eigenvectors and fractionalization of offset DFTs and DCTs," IEEE Trans. Signal Process., vol. 52, no. 7, pp. 2032-2046, Jul. 2004.

[16] F. A. Grünbaum, "The eigenvectors of the discrete Fourier transform: A version of the Hermite functions," J. Math. Anal. Appl., vol. 88, pp. 355-363, 1982.

[17] S. Clary and D. H. Mugler, "Shifted Fourier matrices and their tridiagonal commutors," SIAM J. Matrix Anal. Appl., vol. 24, pp. 809-821, 2003.

[18] S. C. Pei, M. H. Yeh, and C. C. Tseng, "Discrete fractional Fourier transform based on orthogonal projections," IEEE Trans. Signal Process., vol. 47, no. 5, pp. 1335-1348, May 1999.

[19] J. W. Goodman, Introduction to Fourier Optics, 2nd ed. New York: McGraw-Hill, 1996.

[20] C. Candan, M. A. Kutay, and H. M. Ozaktas, "The discrete fractional Fourier transform," IEEE Trans. Signal Process., vol. 48, no. 5, pp. 1329-1337, May 2000.

[21] D. Mendlovic, H. M. Ozaktas, and A. W. Lohmann, "Graded-index fibers, Wigner distribution and the fractional Fourier transform," Appl. Opt., vol. 33, pp. 6188-6193, 1994.

[22] T. Alieva and A. M. Barbe, "Self-fractional Fourier functions and selection of modes," J. Phys. A: Math. Gen., vol. 30, pp. 211-215, 1997.

[23] M. Nazarathy and J. Shamir, "First-order optics: Operator representation for systems with loss or gain," J. Opt. Soc. Amer., vol. 72, no. 10, pp. 1398-1408, Oct. 1982.

[24] M. Nazarathy, A. Hardy, and J. Shamir, "Generalized mode propagation in first-order optical systems with loss or gain," J. Opt. Soc. Amer., vol. 72, no. 10, pp. 1409-1420, Oct. 1982.

[25] K. B. Wolf, "On self-reciprocal functions under a class of integral transforms," J. Math. Phys., vol. 18, pp. 1046-1051, 1977.

[26] J. H. McClellan and T. W. Parks, "Eigenvalue and eigenvector decomposition of the discrete Fourier transform," IEEE Trans. Audio Electroacoust., vol. AE-20, no. 1, pp. 66-74, Mar. 1972. 
[27] N. M. Atakishiyev, L. E. Vicent, and K. B. Wolf, "Continuous versus discrete fractional Fourier transforms," J. Comput. Appl. Math., vol. 107, no. 1, pp. 73-95, Jul. 1999.

[28] T. Alieva and M. J. Bastiaans, "Self-affinity in phase space," J. Opt. Soc. Amer. A, vol. 17, no. 4, pp. 756-761, Apr. 2000

[29] T. Alieva and M. L. Calvo, "Fractionalization of the linear cyclic transforms," J. Opt. Soc. Amer. A, vol. 17, no. 12, pp. 2330-2338, Dec. 2000.

[30] M. T. Hanna, N. P. A. Seif, and W. A. E. M. Ahmed, "Hermite-Gaussian-like eigenvectors of the discrete Fourier transform matrix based on the direct utilization of the orthogonal projection matrices on its eigenspaces," IEEE Trans. Signal Process., vol. 54, no. 7, pp. 2815-2819, Jul. 2006.

[31] M. J. Bastiaans and T. Alieva, "First-order optical systems with unimodular eigenvalues," J. Opt. Soc. Amer. A, vol. 23, no. 8, pp. 1875-1883, Aug. 2006.

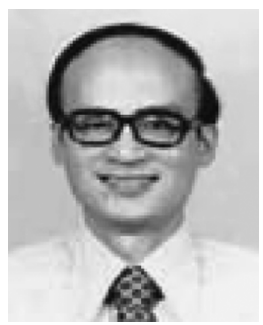

Soo-Chang Pei (SM'89-F'00) was born in Soo-Auo, Taiwan, R.O.C., in 1949. He received the B.S.E.E. degree from the National Taiwan University (NTU), Taipei, Taiwan, R.O.C., in 1970, and the M.S.E.E. and $\mathrm{Ph} . \mathrm{D}$. degrees from the University of California, Santa Barbara (UCSB), in 1972 and 1975, respectively.

He was an Engineering Officer in the Chinese Navy Shipyard from 1970 to 1971 . From 1971 to 1975, he was a Research Assistant with UCSB. He was the Professor and Chairman in the Electrical Engineering Department, Tatung Institute of Technology and NTU, from 1981 to 1983 and 1995 to 1998 , respectively. Presently, he is the Dean of Electrical Engineering and Computer Science College and the Professor of
Electrical Engineering Department, NTU. His research interests include digital signal processing, image processing, optical information processing, and laser holography.

Dr. Pei is a member of Eta Kappa Nu and the Optical Society of America. He received the National Sun Yet-Sen Academic Achievement Award in Engineering in 1984, the Distinguished Research Award from the National Science Council from 1990 to 1998, the outstanding Electrical Engineering Professor Award from the Chinese Institute of Electrical Engineering in 1998, the Academic Achievement Award in Engineering from the Ministry of Education in 1998, the Pan Wen-Yuan Distinguished Research Award in 2002, and the National Chair Professor Award from the Ministry of Education in 2002. He was President of the Chinese Image Processing and Pattern Recognition Society in Taiwan from 1996 to 1998. He became an IEEE Fellow in 2000 for contributions to the development of digital eigenfilter design, color image coding and signal compression, and electrical engineering education in Taiwan.

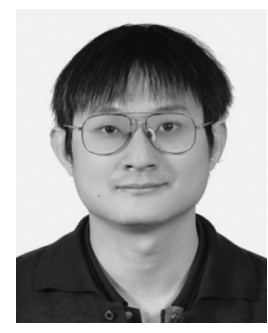

Jian-Jiun Ding was born in 1973 in Taiwan, R.O.C. He received the B.S., M.S., and Ph.D. degrees in electrical engineering from the National Taiwan University (NTU), Taipei, Taiwan, R.O.C., in 1995, 1997, and 2001, respectively.

During 2001 to 2005, he was a Postdoctoral Researcher in the Department of Electrical Engineering of NTU. He is currently an Assistant Professor in the Department of Electrical Engineering, NTU. His current research areas include time-frequency analysis, fractional Fourier transforms, linear canonical transforms, image processing, orthogonal polynomials, fast algorithms, quaternion algebra, pattern recognition, filter design, etc. 